\title{
Avaliação físico-química de bolo de chocolate com coberturas comestíveis à base de gelatina, ácido esteárico, amido modificado ou cera de carnaúba
}

\author{
Physical and chemical evaluation of chocolate cake covered with gelatin, \\ stearic acid, modified starch or "carnaúba" wax edible icing
}

\author{
Cibele Cristina OSAWA ${ }^{1}$, Luciana Cristina Brigatto FONTES ${ }^{1}$, \\ Eduardo Henrique Walter MIRANDA ${ }^{1}$, Yoon Kil CHANG ${ }^{1}$, Caroline Joy STEEL ${ }^{1 *}$
}

\section{Resumo}

Coberturas comestíveis biodegradáveis são uma alternativa às embalagens sintéticas, que causam preocupações ambientais. Este trabalho avaliou o efeito de diferentes tipos de coberturas sobre propriedades físico-químicas de bolo de chocolate durante a estocagem, em comparação com bolo sem cobertura (CO) e bolo sem cobertura embalado em polipropileno (EMB). As seguintes coberturas foram aplicadas sobre os bolos: $10 \%$ gelatina (GE), 10\% gelatina com 10\% ácido esteárico (GE + AE), 18\% cera de carnaúba (CE), 10\% amido modificado (AM) e fondant (FO). Os bolos foram avaliados durante 10 dias de estocagem. FO e EMB apresentaram menor perda de massa, enquanto todos os demais tratamentos apresentaram valores superiores a CO. GE, GE + AE e EMB apresentaram a menor redução da atividade de água, enquanto CE e CO apresentaram a maior redução. As superfícies dos bolos recobertos estavam mais duras que as de CO e EMB. Os maiores valores para dureza e mastigabilidade foram encontrados para CE e CO e os menores, para EMB, GE e GE + AE. Em relação à cor, GE + AE foi diferente dos demais tratamentos, devido à presença do ácido esteárico. Os resultados indicam que a perda de massa dos bolos pode ser atribuída também à perda de água das coberturas.

Palavras-chave: bolo de chocolate; coberturas comestíveis; perda de massa; atividade de água; textura; cor.

\begin{abstract}
Biodegradable edible icing or frosting is an alternative to synthetic packaging that causes environmental concerns. This work evaluated the effect of different types of frosting on the physical-chemical properties of chocolate cake during storage in comparison to cakes without icing or frosting $(\mathrm{C})$ and cakes without frosting packed in polypropylene (CP). The following frostings were applied to the cakes: $10 \%$ gelatin (GE), $10 \%$ gelatin with $10 \%$ stearic acid (GE+SA), $18 \%$ "carnaúba" wax (CW), $10 \%$ modified starch (MS), and fondant (FO). The cakes were analyzed during 10 days of storage. FO and CP presented the lowest mass loss while all other treatments presented higher values than $\mathrm{C}$. $\mathrm{GE}, \mathrm{GE}+\mathrm{SA}$, and $\mathrm{CP}$ presented the lowest reduction of water activity while $\mathrm{CW}$ and $\mathrm{C}$ presented the highest reduction. The surfaces of the frosted cakes were harder than $\mathrm{C}$ and $\mathrm{CP}$. The highest values for hardness and chewiness were found for $\mathrm{CW}$ and $\mathrm{C}$ and the lowest for $\mathrm{CP}$, $\mathrm{GE}$, and GE+SA. With respect to color, GE+SA was different from the remaining cakes due to the presence of the stearic acid. The results indicate that the mass loss of the cakes could also be attributed to the loss of water from the frostings.
\end{abstract}

Keywords: chocolate cake; edible coatings; mass loss; water activity; texture; color.

\section{Introdução}

Os bolos prontos para o consumo vêm adquirindo crescente importância no mercado de produtos de panificação no Brasil. Chudzikiewicz (2005) observou que bolos e tortas são a segunda categoria de produtos que motivam a compra nas padarias, depois do pão. Também, nos últimos anos, muitas empresas que já trabalhavam com pães, biscoitos e torradas ingressaram no mercado de bolos, a fim de diversificar sua linha de produtos (PAVANELLI; CICHELLO; PALMA, 2000).

A qualidade dos bolos é determinada por características essenciais, como: textura macia, que deve permanecer inalterada ao longo da vida de prateleira do produto; superfície uniforme; homogeneidade do miolo; volume adequado; palatabilidade e sabor agradável; e facilidade de processamento. Estes atributos estão diretamente relacionados à qualidade dos ingredientes e seu balanceamento, ao tipo de processamento (PAVANELLI; CICHELLO; PALMA, 2000), à embalagem e às condições de estocagem. A vida útil de bolos está compreendida entre $1 \mathrm{e}$ 4 semanas ou mais, sendo limitada por estes fatores de qualidade, incluindo a atividade de água (GÉLINAS; ROY; GUILLET, 1999).

A perda de umidade resulta em alterações indesejáveis na textura, enquanto que o ganho de umidade pode ocasionar o desenvolvimento microbiano (POTTER; HOTCHKISS, 1998). Para prevenir a perda de umidade, os produtos são geralmente

Recebido para publicação em 12/7/2007

Aceito para publicação em 19/10/2007 (002677)

Departamento de Tecnologia de Alimentos, Faculdade de Engenharia de Alimentos, Universidade Estadual de Campinas - UNICAMP, Rua: Monteiro Lobato, 80, CP 6121, CEP 13083-862, Campinas - SP, Brasil,E-mail: cjsteel@yahoo.com

${ }^{*}$ A quem a correspondência deve ser enviada 
embalados em filmes plásticos que promovam boa barreira ao vapor d'água. Assim, é assegurado que o nível correto de umidade é retido no produto (DENDY; DOBRASZCZYK, 2001; JONES, 1996).

Entretanto, a questão do impacto ambiental ocasionado pelo acúmulo das embalagens sintéticas tem promovido pesquisas sobre materiais biodegradáveis e, particularmente, sobre biofilmes (CUQ et al., 1997).

Os biofilmes incluem os filmes propriamente ditos, usados como embalagens, e as coberturas comestíveis, aplicadas diretamente sobre os alimentos. A cobertura é uma fina camada de material aplicado e formado diretamente na superfície do produto, enquanto que o filme é pré-formado separadamente e aplicado posteriormente sobre o produto (KROCHTA; MULDER-JOHNSTON, 1997).

O interesse em barreiras comestíveis para o controle da transferência de umidade em alimentos é justificado pela necessidade de se manter as características de qualidade durante a estocagem, aliada à conscientização com o volume de embalagens sintéticas descartadas (KESTER; FENNEMA, 1986; KOELSCH; LABUZA, 1992). Além disso, podem ser introduzidos aditivos às coberturas, tais como antioxidantes, aromas e agentes antimicrobianos, melhorando com isso a integridade do produto (KESTER; FENNEMA, 1986).

Os biopolímeros mais utilizados na elaboração de filmes e coberturas comestíveis são as proteínas (gelatina, caseína, ovoalbumina, glúten de trigo, zeína e proteínas miofibrilares), os polissacarídios (amido e seus derivados, pectina, celulose e seus derivados, alginato e carragena) e os lipídios (monoglicerídios acetilados, ácido esteárico, cera e ésteres de ácido graxo) ou a combinação deles (CUQ; GUILBERT, 1995).

No Brasil, a gelatina é produzida em abundância, a baixo custo e com propriedades funcionais adequadas para a fabricação de biofilmes. Entretanto, coberturas à base de gelatina são altamente permeáveis ao vapor d'água, devido à natureza hidrofílica da proteína (BALDWIN et al., 1997).

A incorporação de ácidos graxos às coberturas comestíveis pode promover uma redução na permeabilidade ao vapor d'água (PÉROVAL et al., 2002; WONG et al., 1992). No entanto, produz coberturas opacas (GALLO et al., 2000; GONTARD et al., 1994; PÉROVAL et al., 2002; YANG; PAULSON, 2000), pouco flexíveis e com sabor residual que pode influenciar as características sensoriais do produto (GALLO et al., 2000). O uso de ceras comestíveis é uma outra opção de cobertura, devido à sua natureza hidrofóbica (YANG; PAULSON, 2000).

$\mathrm{Na}$ formulação das coberturas, podem-se combinar proteínas ou polissacarídios com emulsões lipídicas ou camadas lipídicas. Os lipídios atuam na redução da transferência de vapor d'água e as proteínas ou os polissacarídios são componentes estruturais (GONTARD et al., 1994) e excelentes barreiras a gases, aromas e lipídios (YANG; PAULSON, 2000).

Filmes e coberturas comestíveis podem ser aplicados em diversos tipos de alimentos. No entanto, as pesquisas concentramse na área de frutas, vegetais e carnes (CUQ et al., 1995). Não foram encontrados trabalhos envolvendo seu uso em produtos de panificação.

O objetivo do presente trabalho foi estudar o efeito do uso de coberturas à base de gelatina, gelatina com ácido esteárico, amido modificado e emulsão de cera de carnaúba comercial sobre propriedades físico-químicas de bolo sabor chocolate durante a estocagem à temperatura ambiente por 10 dias. Foram usados como controles bolos recobertos com cobertura à base de fondant (padrão do mercado), bolos sem cobertura e sem embalagem e bolos sem cobertura embalados em sacos de polipropileno.

\section{Material e métodos}

\subsection{Materiais}

- Pré-mistura para bolo de chocolate sem conservantes (Zelândia Bolo Inglês Chocolate, Emulzint, Brasil); ovos (Fazendão Ovos, Brasil) e leite em pó integral (Alibra, Brasil), para o preparo dos bolos;

- Gelatina alimentícia de origem suína, tipo A, 250P bloom, 30 mesh, viscosidade $42 \mathrm{mPs}$ (Gelita, Brasil); ácido esteárico (Merck, Alemanha); amido modificado (Amidomax 4800, Cargill, Brasil) e emulsão de cera de carnaúba $18 \%$ (Meghwax ECF 124, Megh, Brasil), cujo uso é recomendado como cobertura de mamão, manga, melão, maçã e limão (MEGH, 2005), para o preparo das coberturas;

- Fondant (Fleischmann, Brasil), chocolate em pó solúvel e margarina para o preparo da cobertura padrão do mercado;

- Formas de alumínio descartáveis (210 x $100 \times 45$ mm) para o forneamento e contenção dos bolos; e

- Embalagens de polipropileno cast ( 345 X 163 mm; $22 \mu \mathrm{m}$ de espessura) pregueadas na base e amarris de arame plastificado para o acondicionamento de um dos tratamentos dos bolos.

\subsection{Métodos}

\section{Preparo dos bolos}

Os bolos foram preparados de acordo com as instruções do fornecedor, nas seguintes proporções: $1 \mathrm{~kg}$ de pré-mistura, $250 \mathrm{~g}$ de ovos, $20 \mathrm{~g}$ de leite em pó integral e $250 \mathrm{~g}$ de água (EMULZINT, 2005). Os ingredientes foram misturados em batedeira industrial tipo BA n ${ }^{\circ} 1556$ (Siam Útil, Brasil), durante 6 minutos, iniciando-se com o batimento dos ovos em rotação alta, seguido da adição da água, do leite em pó e da pré-mistura em rotação baixa. Porções de $250 \mathrm{~g}$ de massa de bolo foram colocadas nas fôrmas de alumínio e forneadas a $160^{\circ} \mathrm{C}$, durante 55 minutos. Após o forneamento, os bolos foram mantidos nas fôrmas de alumínio e resfriados à temperatura ambiente para a aplicação das coberturas no mesmo dia. Foram preparados 200 bolos, com volume específico médio de $2,6 \pm 0,3 \mathrm{~cm}^{3} / \mathrm{g}$, escolhendo-se de forma aleatória os bolos destinados a cada tratamento. 
Preparo das coberturas

Gelatina (GE)

A cobertura à base de gelatina foi preparada hidratando-se $10 \mathrm{~g}$ de gelatina em $100 \mathrm{~mL}$ de água destilada, por 1 hora à temperatura ambiente. Após este período, a gelatina foi solubilizada a $95^{\circ} \mathrm{C}$ em banho termostático TE-052 Dubnoff Fibralimentar (Tecnal, Brasil) por 10 minutos.

\section{Gelatina + ácido esteárico (GE + AE)}

A gelatina foi hidratada e solubilizada conforme descrito no item Gelatina (GE). Em seguida, adicionaram-se $10 \mathrm{~g}$ de ácido esteárico à solução de gelatina, mantendo-se a mistura no banho termostático a $95{ }^{\circ} \mathrm{C}$, para garantir a fusão completa do ácido esteárico. Finalmente, a mistura foi homogeneizada utilizandose agitador modelo $713 \mathrm{~T}$ (Fisatom, Brasil), a $6.000 \mathrm{rpm}$, até a formação de uma emulsão.

Amido modificado (AM)

A cobertura à base de amido modificado foi preparada adicionando-se $10 \mathrm{~g}$ de amido modificado a $100 \mathrm{~mL}$ de água destilada e aquecendo-se em banho termostático a $95^{\circ} \mathrm{C}$, até a gelatinização do amido, observada pelo aumento da viscosidade.

Emulsão de cera de carnaúba (CE)

Utilizou-se diretamente o produto comercial emulsão de cera de carnaúba 18\% (Meghwax ECF 124, Megh, Brasil).

\section{Fondant (FO)}

A cobertura de fondant foi preparada utilizando-se $1 \mathrm{~kg}$ de fondant, $250 \mathrm{~g}$ de chocolate em pó solúvel e $250 \mathrm{~g}$ de margarina. A mistura foi aquecida em banho-maria até atingir o ponto de fusão da margarina.

\section{Aplicação das coberturas}

Os bolos receberam a aplicação das coberturas preparadas conforme o item "Preparo das coberturas" sobre a superfície superior, sem serem retirados das fôrmas de alumínio descartáveis. As coberturas foram aplicadas com o auxílio de um pincel para confeitaria, em uma única aplicação para os bolos revestidos com amido modificado (AM) e fondant (FO), e em bicamada para os demais tratamentos com cobertura.

As quantidades das coberturas aplicadas foram: aproximadamente $20 \mathrm{~g}$ de cobertura de gelatina (GE), $9 \mathrm{~g}$ de cobertura de gelatina com ácido esteárico $(\mathrm{GE}+\mathrm{AE}), 15 \mathrm{~g}$ de cobertura de amido modificado (AM), 8 g de emulsão de cera de carnaúba comercial (CE) e $50 \mathrm{~g}$ de fondant (FO), por camada, para cada bolo. As quantidades utilizadas foram suficientes para recobrir completamente a superfície do bolo.

As coberturas foram aplicadas nas superfícies dos bolos à temperatura ambiente para $\mathrm{CE}$, a $45^{\circ} \mathrm{C}$ para $\mathrm{GE}$ e, imediatamente após a retirada do banho, para GE + AE, AM e FO.
A segunda camada foi aplicada cerca de 15 minutos após a aplicação da primeira, exceto para o tratamento com CE, para o qual foi após 1 hora.

\section{Estocagem dos bolos}

Os bolos foram estocados à temperatura ambiente, durante 10 dias. As condições climáticas de Campinas durante o período foram: temperatura mínima média de $17,9 \pm 1,4^{\circ} \mathrm{C}$, temperatura máxima média de $28,2 \pm 3,4^{\circ} \mathrm{C}$ e umidade relativa média de 93,2 \pm 5,6\% (AGRITEMPO, 2005; CEPAGRI, 2005).

\section{Análise dos bolos}

Os bolos foram monitorados em relação à perda de massa, atividade de água, textura e cor, em intervalos de tempo determinados. Os bolos com a cobertura à base de fondant (FO), o tratamento incluindo bolos sem cobertura e sem embalagem (CO) e o tratamento incluindo bolos sem cobertura, embalados em sacos de polipropileno (EMB) foram utilizados como controles.

\section{Perda de massa}

A determinação da perda de massa foi realizada pesando-se os bolos em balança analítica (Quimis, Brasil), em quadruplicata para cada tratamento, nos dias $0,1,3,4,5,6,7$ e 10 de estocagem. Considerou-se a porcentagem de perda de massa em relação à massa inicial.

\section{Atividade de água}

A atividade de água foi determinada no meio do miolo de 4 fatias distintas de bolo para cada tratamento. As análises foram realizadas nos dias $0,1,3,4,7$ e 10 de estocagem, utilizando-se o equipamento Aqualab, modelo 3 TE (Braseq, Brasil).

\section{Textura}

A textura dos bolos foi avaliada através da força de perfuração na sua superfície e do perfil de textura (TPA) no seu interior. As análises foram realizadas nos dias 0, 1, 3, 4, 7 e 10 de estocagem, utilizando-se o texturômetro de bancada TAXT2 (Stable Micro Systems, UK).

Força de perfuração: método recomendado para "chocolate filled caramel", segundo o manual do equipamento; probe de alumínio $\mathrm{P} / 2$, haste curta, mesa como base; velocidade préteste de $1,0 \mathrm{~mm} / \mathrm{s}$; velocidade de teste de $0,5 \mathrm{~mm} / \mathrm{s}$; velocidade pós-teste de $10,0 \mathrm{~mm} / \mathrm{s}$; distância de $4,0 \mathrm{~mm}$. A análise foi realizada em 2 bolos inteiros para cada tratamento, escolhendose os pontos de perfuração na região central. Foram utilizadas 5 repetições.

Perfil de textura (TPA): avaliado em 2 fatias centrais sobrepostas de 1,5 cm cada, cortadas com fatiadora elétrica, utilizando-se probe de alumínio $\mathrm{P} / 100$; velocidade pré-teste de $5,0 \mathrm{~mm} / \mathrm{s}$; velocidade de teste de $2,0 \mathrm{~mm} / \mathrm{s}$; velocidade pós-teste de 5,0 mm/s; distância de 10,0 mm (SARMIENTO-CONSOLE, 1998). Avaliaram-se os parâmetros dureza, coesividade e mastigabilidade. Foram utilizadas 5 repetições. 
Cor

A análise instrumental de cor foi realizada diretamente sobre a superfície do bolo, nos dias 1, 3 e 4 de estocagem, em triplicata, utilizando-se o colorímetro ColorQUEST II (HunterLab, EUA). As condições de mensuração foram as seguintes: calibração de Refletância Especular Excluída (RSEX), iluminante $\mathrm{D}_{65}$, ângulo de observação de $10^{\circ}$. O espaço de cor utilizado foi o $L^{\star} C^{\star} h$. A diferença de cor $\left(\Delta \mathrm{E}^{\star}\right)$ de cada tratamento foi determinada ao longo da estocagem em relação ao bolo do primeiro dia.

\section{Análises estatísticas}

Foram realizadas comparações entre as médias, através da Análise de Variância (ANOVA) e Teste de Tukey, ao nível de $5 \%$ de significância, utilizando o software SAS for Windows, versão 8.2 (COUNCIL, 1985).

\section{Resultados e discussão}

\subsection{Perda de massa}

A perda de massa dos bolos ficou entre 0,2 e $12,2 \%$ até o $10^{\circ}$ dia de estocagem (Tabela 1). Observa-se que o tratamento mais eficaz no controle da perda de massa foi o EMB. Este comportamento era esperado, pois a embalagem de polipropileno é uma boa barreira relativa ao vapor d'água (SARANTÓPOULOS et al., 2002).

Dentre os tratamentos com coberturas, o FO foi o único que inibiu a perda de massa, tendo valores menores que o $\mathrm{CO}$ em todos os dias avaliados. Nos demais bolos recobertos, ocorreu uma perda de massa superior ao CO. O fondant possui um alto teor de lipídios e açúcar, com um menor teor de umidade (aproximadamente 10\%) quando comparado às outras coberturas.

Os tratamentos GE e AM foram os que apresentaram as maiores perdas de massa ao longo da estocagem. Esta perda de massa pode ser atribuída à perda de água das próprias coberturas, que possuíam elevado teor de água em sua composição (aproximadamente 90\%) e totalizavam cerca de 4 a $14 \%$ da massa total do bolo recoberto. Este fenômeno é observado no processo de secagem para a formação de coberturas (GONTARD, 1994). Além disto, pode ter ocorrido uma formação incompleta ou desuniforme da cobertura sobre a superfície do bolo. A porosidade e a superfície desuniforme dos bolos podem ter absorvido a cobertura, que no momento da aplicação estava líquida, com viscosidade próxima à da água.

Os tratamentos $\mathrm{GE}+\mathrm{AE}$ e $\mathrm{CE}$, com comportamentos intermediários, a partir do $5^{\circ}$ dia de estocagem, não apresentaram diferenças significativas. Mesmo apresentando propriedades hidrofóbicas, as perdas de massa destes tratamentos foram superiores ao CO. Isto pode também ser atribuído ao elevado teor de água (aproximadamente 80\%) nestas coberturas.

\subsection{Atividade de água}

A atividade de água $\left(\mathrm{a}_{\mathrm{a}}\right)$ dos bolos variou de 0,716 a 0,918 , durante a estocagem (Tabela 2). Os tratamentos que apresentaram os maiores valores de $\mathrm{a}_{\mathrm{a}}$ no $10^{\circ}$ dia de estocagem foram $\mathrm{GE}$, $\mathrm{EMB}$ e $\mathrm{GE}+\mathrm{AE}$, mantendo as suas características mais próximas às do bolo recém-processado. Estes dados reforçam a hipótese de que a perda de massa dos bolos com coberturas de alto teor de água está relacionada à perda de umidade das próprias coberturas para o ambiente. Além disto, pode-se considerar que a maior $\mathrm{a}_{\mathrm{a}}$ destes bolos esteja também associada à migração de água da cobertura para o miolo do bolo.

Os tratamentos $\mathrm{CO}$ e CE tiveram os menores valores de $\mathrm{a}_{\mathrm{a}}$ no $10^{\circ}$ dia de estocagem. O CO não possuía cobertura, nem embalagem, perdendo umidade diretamente para o ambiente, reduzindo-se a a do bolo. No tratamento CE, ocorreu uma rápida absorção da cobertura que progressivamente foi apresentando rachaduras de maior tamanho, facilitando a perda de umidade do miolo ao longo da estocagem.

\subsection{Textura}

\section{Perfuração}

Os resultados de força de perfuração encontram-se na Tabela 3. Os bolos do tratamento CO apresentaram um amolecimento da crosta entre o dia do processamento e o $1^{\circ}$ dia de estocagem. Este amolecimento está associado à migração de umidade do miolo do bolo para sua crosta, ocasionando o equilíbrio de umidade no produto.

No $1^{\circ}$ dia de estocagem, AM e CE apresentaram superfície com valores de força de perfuração em uma faixa similar a $\mathrm{CO}$ e $\mathrm{EMB}$, enquanto que as demais coberturas tornaram a superfície

Tabela 1. Perda de massa, em porcentagem, dos bolos ao longo da estocagem.

\begin{tabular}{lccccccc}
\hline Tratamento & Dia 1 & Dia 3 & Dia 4 & Dia 5 & Dia 6 & Dia 7 \\
\hline $\mathrm{CO}$ & $-0,1 \pm 0,0^{\mathrm{eF}}$ & $2,1 \pm 0,1^{\mathrm{dE}}$ & $3,3 \pm 0,2^{\mathrm{eD}}$ & $4,0 \pm 0,2^{\mathrm{dC}}$ & $4,7 \pm 0,3^{\mathrm{dBC}}$ & $5,0 \pm 0,3^{\mathrm{dB}}$ & $6,6 \pm 0,5^{\mathrm{dA}}$ \\
$\mathrm{EMB}$ & $0,0 \pm 0,0^{\mathrm{eD}}$ & $0,0 \pm 0,0^{\mathrm{fCD}}$ & $0,1 \pm 0,0^{\mathrm{gBC}}$ & $0,1 \pm 0,0^{\mathrm{BBC}}$ & $0,1 \pm 0,0^{\mathrm{fB}}$ & $0,1 \pm 0,0^{\mathrm{fB}}$ & $0,2 \pm 0,0^{\mathrm{fA}}$ \\
$\mathrm{GE}$ & $1,6 \pm 0,1^{\mathrm{aF}}$ & $5,8 \pm 0,5^{\mathrm{aE}}$ & $7,3 \pm 0,5^{\mathrm{aD}}$ & $8,6 \pm 0,5^{\mathrm{aC}}$ & $9,5 \pm 0,5^{\mathrm{aBC}}$ & $10,1 \pm 0,5^{\mathrm{aB}}$ & $12,2 \pm 0,6^{\mathrm{aA}}$ \\
$\mathrm{GE}+\mathrm{AE}$ & $1,3 \pm 0,1^{\mathrm{bF}}$ & $4,2 \pm 0,2^{\mathrm{bE}}$ & $5,3 \pm 0,3^{\mathrm{cD}}$ & $6,2 \pm 0,3^{\mathrm{cC}}$ & $6,9 \pm 0,3^{\mathrm{cB}}$ & $7,3 \pm 0,3^{\mathrm{cB}}$ & $8,8 \pm 0,3^{\mathrm{cA}}$ \\
$\mathrm{CE}$ & $0,5 \pm 0,1^{\mathrm{cF}}$ & $3,2 \pm 0,2^{\mathrm{cE}}$ & $4,6 \pm 0,2^{\mathrm{dD}}$ & $5,5 \pm 0,3^{\mathrm{cC}}$ & $6,2 \pm 0,4^{\mathrm{cBC}}$ & $6,6 \pm 0,5^{\mathrm{cB}}$ & $8,3 \pm 0,7^{\mathrm{cA}}$ \\
$\mathrm{AM}$ & $1,6 \pm 0,1^{\mathrm{aE}}$ & $5,4 \pm 0,3^{\mathrm{aD}}$ & $6,6 \pm 0,4^{\mathrm{bC}}$ & $7,6 \pm 0,5^{\mathrm{bBC}}$ & $8,3 \pm 0,6^{\mathrm{bB}}$ & $8,7 \pm 0,6^{\mathrm{bB}}$ & $10,3 \pm 0,7^{\mathrm{bA}}$ \\
$\mathrm{FO}$ & $0,2 \pm 0,0^{\mathrm{dE}}$ & $0,7 \pm 0,0^{\mathrm{eD}}$ & $1,0 \pm 0,1^{\mathrm{fCD}}$ & $1,2 \pm 0,1^{\mathrm{eC}}$ & $1,4 \pm 0,2^{\mathrm{eBC}}$ & $1,7 \pm 0,3^{\mathrm{eB}}$ & $2,2 \pm 0,4^{\mathrm{eA}}$ \\
\hline
\end{tabular}

$\mathrm{GE}=$ gelatina; $\mathrm{GE}+\mathrm{AE}=$ gelatina com ácido esteárico; $\mathrm{AM}=$ amido modificado; $\mathrm{CE}=$ cera de carnaúba; $\mathrm{FO}=$ fondant $\mathrm{CO}=$ sem cobertura; e $\mathrm{EMB}=$ embalado. Médias seguidas de mesma letra minúscula na coluna e maiúscula na linha não diferem entre si ao nível de $5 \%$ de significância. 
do bolo mais dura. A cobertura de fondant foi a que apresentou o maior valor de força de perfuração.

Em geral, ocorreu um endurecimento da superfície de todos os tratamentos ao longo da estocagem, com exceção do bolo embalado. O EMB apresentou o menor valor de força de perfuração durante a estocagem, sendo que entre o $1^{\circ}$ e o $3^{\circ}$ dia ocorreu um equilíbrio de umidade entre o miolo do bolo, a crosta e o microambiente da embalagem. Este fenômeno também é relatado por Sluimer (2005).

No $10^{\circ}$ dia de estocagem, os tratamentos AM e CE apresentaram valores de força de perfuração da superfície similares ao $\mathrm{CO}$, mas inferiores às demais coberturas e superiores ao EMB.

\section{Dureza}

Os resultados de dureza encontram-se na Tabela 4. Os tratamentos EMB, GE, GE + AE e FO foram os que apresentaram menores valores de dureza, a partir do $4^{\circ}$ dia de estocagem. No $10^{\circ} \mathrm{dia}$, sua dureza variou de $5.628 \mathrm{~g}(\mathrm{EMB})$ a $7.606 \mathrm{~g}(\mathrm{FO})$. Os três primeiros tratamentos foram os que mantiveram valores mais elevados de $\mathrm{a}_{\mathrm{a}}$.

O tratamento $\mathrm{CO}$ apresentou a maior dureza durante a estocagem. A dureza deste tratamento no $10^{\circ}$ dia de estocagem foi mais que o dobro da inicial. Gélinas, Roy e Guillet (1999) encontraram o dobro da dureza inicial em bolos estocados a $21{ }^{\circ} \mathrm{C}$ por 21 dias. A falta de uma barreira (embalagem ou cobertura) neste tratamento conduziu à perda de umidade do próprio bolo. Paralelamente, deve-se considerar o efeito da retrogradação (SLUIMER, 2005).

No entanto, nos tratamentos AM e CE, as coberturas não retardaram o aumento da dureza dos bolos da mesma forma que as demais coberturas ou a embalagem.

\section{Coesividade}

Os resultados de coesividade encontram-se na Tabela 5. Verificou-se que os tratamentos não apresentaram diferença significativa entre si até o $4^{\circ}$ dia de estocagem. Nos demais dias, verificou-se uma ligeira queda na coesividade dos tratamentos

Tabela 2. Atividade de água dos bolos ao longo da estocagem.

\begin{tabular}{lllllll}
\hline Tratamento & Dia 0 & Dia 1 & Dia 3 & Dia 4 & Dia 7 & Dia 10 \\
\hline CO & $0,911 \pm 0,019^{\mathrm{aA}}$ & $0,900 \pm 0,014^{\mathrm{aAB}}$ & $0,844 \pm 0,040^{\mathrm{aB}}$ & $0,759 \pm 0,049^{\mathrm{cC}}$ & $0,760 \pm 0,037^{\mathrm{cC}}$ & $0,716 \pm 0,028^{\mathrm{cC}}$ \\
EMB & $0,911 \pm 0,019^{\mathrm{aA}}$ & $0,843 \pm 0,040^{\mathrm{bB}}$ & $0,857 \pm 0,037^{\mathrm{aB}}$ & $0,830 \pm 0,024^{\mathrm{abcB}}$ & $0,863 \pm 0,016^{\mathrm{abAB}}$ & $0,846 \pm 0,014^{\mathrm{aB}}$ \\
GE & $0,911 \pm 0,019^{\mathrm{aA}}$ & $0,908 \pm 0,014^{\mathrm{aAB}}$ & $0,903 \pm 0,007^{\mathrm{aAB}}$ & $0,897 \pm 0,014^{\mathrm{aABC}}$ & $0,890 \pm 0,004^{\mathrm{aBC}}$ & $0,873 \pm 0,014^{\mathrm{aC}}$ \\
$\mathrm{GE}+\mathrm{AE}$ & $0,911 \pm 0,019^{\mathrm{aA}}$ & $0,876 \pm 0,021^{\mathrm{abAB}}$ & $0,861 \pm 0,024^{\mathrm{aB}}$ & $0,856 \pm 0,040^{\mathrm{aB}}$ & $0,842 \pm 0,026^{\mathrm{abB}}$ & $0,831 \pm 0,004^{\mathrm{abB}}$ \\
AM & $0,911 \pm 0,019^{\mathrm{aA}}$ & $0,888 \pm 0,034^{\mathrm{abAB}}$ & $0,872 \pm 0,016^{\mathrm{aABC}}$ & $0,837 \pm 0,026^{\mathrm{abBDC}}$ & $0,828 \pm 0,025^{\mathrm{abDC}}$ & $0,796 \pm 0,026^{\mathrm{bD}}$ \\
CE & $0,911 \pm 0,019^{\mathrm{aA}}$ & $0,918 \pm 0,005^{\mathrm{aA}}$ & $0,879 \pm 0,023^{\mathrm{aB}}$ & $0,848 \pm 0,035^{\mathrm{abC}}$ & $0,835 \pm 0,039^{\mathrm{abC}}$ & $0,733 \pm 0,028^{\mathrm{cC}}$ \\
FO & $0,911 \pm 0,019^{\mathrm{aA}}$ & $0,878 \pm 0,026^{\mathrm{abAB}}$ & $0,856 \pm 0,027^{\mathrm{aAB}}$ & $0,796 \pm 0,033^{\mathrm{bcBC}}$ & $0,806 \pm 0,025^{\mathrm{bcBC}}$ & $0,797 \pm 0,021^{\mathrm{bD}}$ \\
\hline
\end{tabular}

$\mathrm{GE}$ = gelatina; $\mathrm{GE}+\mathrm{AE}$ = gelatina com ácido esteárico; $\mathrm{AM}=$ amido modificado; $\mathrm{CE}=$ cera de carnaúba; $\mathrm{FO}=$ fondant $\mathrm{CO}=$ sem cobertura; e $\mathrm{EMB}=$ embalado. Médias seguidas de mesma letra minúscula na coluna e maiúscula na linha não diferem entre si ao nível de $5 \%$ de significância.

Tabela 3. Força de perfuração, em g, dos bolos ao longo da estocagem.

\begin{tabular}{lcccccc}
\hline Tratamento & Dia 0 & Dia 1 & Dia 3 & Dia 4 & Dia 7 & Dia 10 \\
\hline CO & $185 \pm 8^{\mathrm{aA}}$ & $70 \pm 7^{\mathrm{deD}}$ & $78 \pm 5^{\mathrm{eD}}$ & $110 \pm 6^{\mathrm{cC}}$ & $109 \pm 3^{\mathrm{dC}}$ & $158 \pm 23^{\mathrm{dB}}$ \\
EMB & - & $65 \pm 6^{\mathrm{efA}}$ & $51 \pm 3^{\mathrm{eB}}$ & $50 \pm 6^{\mathrm{dB}}$ & $55 \pm 5^{\mathrm{eB}}$ & $48 \pm 4^{\mathrm{eB}}$ \\
GE & - & $112 \pm 7^{\mathrm{cD}}$ & $315 \pm 29^{\mathrm{aB}}$ & $314 \pm 37^{\mathrm{aB}}$ & $485 \pm 25^{\mathrm{aA}}$ & $202 \pm 9^{\mathrm{bcC}}$ \\
$\mathrm{GE}+\mathrm{AE}$ & - & $137 \pm 6^{\mathrm{bC}}$ & $196 \pm 14^{\mathrm{bB}}$ & $187 \pm 17^{\mathrm{bB}}$ & $268 \pm 29^{\mathrm{bA}}$ & $243 \pm 26^{\mathrm{aA}}$ \\
$\mathrm{AM}$ & - & $84 \pm 6^{\mathrm{dD}}$ & $106 \pm 8^{\mathrm{dC}}$ & $118 \pm 11^{\mathrm{cC}}$ & $140 \pm 10^{\mathrm{cdB}}$ & $184 \pm 18^{\mathrm{cdA}}$ \\
$\mathrm{CE}$ & - & $55 \pm 7^{\mathrm{fC}}$ & $148 \pm 9^{\mathrm{cA}}$ & $115 \pm 12^{\mathrm{cB}}$ & $122 \pm 6^{\mathrm{dB}}$ & $163 \pm 7^{\mathrm{dA}}$ \\
FO & - & $181 \pm 12^{\mathrm{aB}}$ & $167 \pm 9^{\mathrm{cB}}$ & $117 \pm 7^{\mathrm{cC}}$ & $176 \pm 41^{\mathrm{cB}}$ & $224 \pm 22^{\mathrm{abA}}$ \\
\hline
\end{tabular}

$\mathrm{GE}=$ gelatina $\mathrm{GE}+\mathrm{AE}=$ gelatina com ácido esteárico; $\mathrm{AM}=$ amido modificado; $\mathrm{CE}=$ cera de carnaúba; $\mathrm{FO}=$ fondant $\mathrm{CO}=$ sem cobertura; e $\mathrm{EMB}=$ embalado. $\mathrm{Médias}$ seguidas de mesma letra minúscula na coluna e maiúscula na linha não diferem entre si ao nível de $5 \%$ de significância.

Tabela 4. Dureza, em g, dos bolos ao longo da estocagem.

\begin{tabular}{|c|c|c|c|c|c|c|}
\hline Tratamento & Dia 0 & Dia 1 & Dia 3 & Dia 4 & Dia 7 & Dia 10 \\
\hline $\mathrm{CO}$ & $6.060 \pm 224^{\mathrm{aC}}$ & $5.712 \pm 328^{\mathrm{aC}}$ & $6.204 \pm 693^{\mathrm{aC}}$ & $10.275 \pm 407^{\mathrm{aB}}$ & - & $15.032 \pm 1.001^{\mathrm{aA}}$ \\
\hline EMB & $6.060 \pm 224^{\mathrm{aA}}$ & $4.726 \pm 155^{\mathrm{bC}}$ & $4.392 \pm 459^{c \mathrm{c}}$ & $5.016 \pm 93^{\mathrm{dBC}}$ & $4.886 \pm 272^{\mathrm{cC}}$ & $5.628 \pm 589^{\mathrm{eAB}}$ \\
\hline GE & $6.060 \pm 224^{\mathrm{aA}}$ & $3.582 \pm 430^{\mathrm{cB}}$ & $4.354 \pm 340^{\mathrm{cB}}$ & $4.511 \pm 355^{\mathrm{dB}}$ & $6.468 \pm 664^{\mathrm{bcA}}$ & $6.497 \pm 1.038^{\mathrm{eA}}$ \\
\hline $\mathrm{GE}+\mathrm{AE}$ & $6.060 \pm 224^{\mathrm{aB}}$ & $3.892 \pm 84^{\mathrm{cD}}$ & $4.938 \pm 239^{\mathrm{bcC}}$ & $5.079 \pm 527^{\mathrm{cdC}}$ & $7.004 \pm 515^{\mathrm{bcA}}$ & $7.395 \pm 694^{\mathrm{deA}}$ \\
\hline $\mathrm{AM}$ & $6.060 \pm 224^{\mathrm{aD}}$ & $4.033 \pm 108^{\mathrm{cE}}$ & $6.342 \pm 416^{\mathrm{aCD}}$ & $7.382 \pm 311^{\mathrm{bBC}}$ & $8.222 \pm 715^{\mathrm{bB}}$ & $9.659 \pm 1.075^{\mathrm{cA}}$ \\
\hline $\mathrm{CE}$ & $6.060 \pm 224^{\mathrm{aBC}}$ & $4.601 \pm 215^{\mathrm{bC}}$ & $5.866 \pm 837^{\mathrm{abBC}}$ & $8.018 \pm 306^{\mathrm{bB}}$ & $11.615 \pm 2.535^{\mathrm{aA}}$ & $12.498 \pm 1.336^{\mathrm{bA}}$ \\
\hline $\mathrm{FO}$ & $6.060 \pm 224^{\mathrm{aBC}}$ & $4.985 \pm 281^{\mathrm{bC}}$ & $6.204 \pm 693^{\mathrm{aB}}$ & $5.885 \pm 730^{\mathrm{cBC}}$ & - & $7.606 \pm 798^{\mathrm{dA}}$ \\
\hline
\end{tabular}

$\mathrm{GE}=$ gelatina $\mathrm{GE}+\mathrm{AE}=$ gelatina com ácido esteárico; $\mathrm{AM}=$ amido modificado; $\mathrm{CE}=$ cera de carnaúba; $\mathrm{FO}=$ fondant $\mathrm{CO}=$ sem cobertura; e $\mathrm{EMB}=$ embalado. Médias seguidas de mesma letra minúscula na coluna e maiúscula na linha não diferem entre si ao nível de $5 \%$ de significância. 
analisados. Isto demonstra que os bolos tornam-se um pouco mais propensos à desintegração com o decorrer da estocagem. Gélinas, Roy e Guillet (1999) observaram uma diminuição a um terço do valor inicial na coesividade de bolos estocados a $21{ }^{\circ} \mathrm{C}$ por 21 dias.

\section{Mastigabilidade}

Os resultados de mastigabilidade encontram-se na Tabela 6. Os tratamentos $\mathrm{CO}$ e CE apresentaram os maiores valores de mastigabilidade a partir do $4^{\circ}$ dia de estocagem, sendo também os de maior dureza (Tabela 4). Estes dados estão de acordo com as expressivas reduções de $\mathrm{a}_{\mathrm{a}}$ observadas nestes tratamentos. Os demais tratamentos não apresentaram diferença significativa entre si, observando-se um ligeiro aumento deste atributo ao longo da estocagem.

\subsection{Cor}

As Tabelas 7, 8, 9 e 10 apresentam as variações dos parâmetros de cor $L^{\star} C^{\star} h$ e a diferença de cor $\left(\Delta E^{\star}\right)$ da superfície dos bolos ao longo da estocagem.

Inicialmente, os bolos dos tratamentos $\mathrm{CO}$ e EMB apresentaram uma crosta com luminosidade $\left(\mathrm{L}^{\star}\right)$ média e tonalidade $(\mathrm{h})$ amarronzada de baixa saturação $\left(C^{\star}\right)$ relativa no espaço $L^{\star} C^{\star} h$. $\mathrm{O}$ tratamento $\mathrm{AM}$ foi o único que manteve os atributos de cor similares ao $\mathrm{CO}$ e ao EMB no $1^{\circ}$ dia de estocagem. Os tratamentos GE, CE e FO apresentaram coloração similar, com saturação de cor ainda mais baixa que a dos bolos sem cobertura. Esta redução na saturação pode ser considerada uma característica sensorial desejável em bolos de chocolate, pois os aproxima mais à coloração típica do chocolate escuro. $\mathrm{O}$ tratamento GE + AE resultou em uma cobertura com luminosidade maior (superfície mais clara) e tonalidade mais amarelada, descaracterizando a coloração típica do chocolate escuro. Isto pode ser atribuído à presença do ácido esteárico na composição da cobertura.

Entre o $1^{\circ}$ e o $3^{\circ}$ dia de estocagem, os bolos dos tratamentos sem cobertura (CO e EMB), assim como GE e GE + AE mostraram uma redução da luminosidade e um aumento da saturação, mas sem alteração significativa da tonalidade. A superfície destes bolos passou a apresentar uma coloração mais carregada ou profunda (deep) (MINOLTA, 1994). No tratamento FO, a diferença de cor esteve associada à mudança da tonalidade, bem como à redução da luminosidade, mas a saturação não variou. A coloração da superfície do bolo do tratamento CE teve uma mudança em todos os seus atributos. Os tratamentos GE + AE e AM foram os que tiveram a menor variação de cor ao longo da estocagem. A variação de cor do tratamento AM ao longo da estocagem esteve associada principalmente à redução de sua luminosidade. Este escurecimento da superfície do bolo de chocolate pode ser considerado um atributo sensorial desejável. Além disto, no $4^{\circ}$ dia de estocagem, AM foi o tratamento que possuía a coloração mais parecida com a dos bolos sem cobertura no $1^{\circ}$ dia.

\section{Conclusões}

As coberturas apresentaram efeitos distintos nas características do bolo, podendo prolongar a vida de prateleira ou influenciar negativamente as propriedades do produto. A embalagem de polipropileno proporcionou as melhores características físico-químicas do bolo ao longo da estocagem. Apesar disto, algumas coberturas mostraram-se como opções potenciais para a conservação de bolos.

Tabela 5. Coesividade dos bolos ao longo da estocagem.

\begin{tabular}{lllllcc}
\hline Tratamento & Dia 0 & Dia 1 & Dia 3 & Dia 4 & Dia 7 & Dia 10 \\
\hline CO & $0,30 \pm 0,02^{\mathrm{aA}}$ & $0,37 \pm 0,01^{\mathrm{aA}}$ & $0,30 \pm 0,01^{\mathrm{bA}}$ & $0,32 \pm 0,01^{\mathrm{aA}}$ & - & $0,32 \pm 0,01^{\mathrm{aA}}$ \\
$\mathrm{EMB}$ & $0,30 \pm 0,02^{\mathrm{aAB}}$ & $0,38 \pm 0,01^{\mathrm{aA}}$ & $0,34 \pm 0,02^{\mathrm{aAB}}$ & $0,25 \pm 0,15^{\mathrm{aB}}$ & $0,30 \pm 0,02^{\mathrm{aAB}}$ & $0,29 \pm 0,01^{\mathrm{abcAB}}$ \\
$\mathrm{GE}$ & $0,30 \pm 0,02^{\mathrm{aBC}}$ & $0,36 \pm 0,03^{\mathrm{aA}}$ & $0,31 \pm 0,03^{\mathrm{abB}}$ & $0,26 \pm 0,02^{\mathrm{aCD}}$ & $0,27 \pm 0,01^{\mathrm{abBCD}}$ & $0,24 \pm 0,03^{\mathrm{cD}}$ \\
$\mathrm{GE}+\mathrm{AE}$ & $0,30 \pm 0,02^{\mathrm{aBC}}$ & $0,39 \pm 0,03^{\mathrm{aA}}$ & $0,33 \pm 0,01^{\mathrm{abB}}$ & $0,29 \pm 0,02^{\mathrm{aBC}}$ & $0,27 \pm 0,01^{\mathrm{abC}}$ & $0,26 \pm 0,03^{\mathrm{bcC}}$ \\
$\mathrm{AM}$ & $0,30 \pm 0,02^{\mathrm{aBC}}$ & $0,37 \pm 0,01^{\mathrm{aA}}$ & $0,32 \pm 0,01^{\mathrm{abB}}$ & $0,33 \pm 0,02^{\mathrm{aB}}$ & $0,24 \pm 0,01^{\mathrm{bD}}$ & $0,26 \pm 0,03^{\mathrm{bcCD}}$ \\
$\mathrm{CE}$ & $0,30 \pm 0,02^{\mathrm{aB}}$ & $0,38 \pm 0,03^{\mathrm{aA}}$ & $0,34 \pm 0,04^{\mathrm{aAB}}$ & $0,32 \pm 0,02^{\mathrm{aAB}}$ & $0,29 \pm 0,04^{\mathrm{aB}}$ & $0,31 \pm 0,03^{\mathrm{abB}}$ \\
FO & $0,30 \pm 0,02^{\mathrm{aAB}}$ & $0,34 \pm 0,06^{\mathrm{aA}}$ & $0,30 \pm 0,01^{\mathrm{bAB}}$ & $0,27 \pm 0,02^{\mathrm{aB}}$ & - & $0,30 \pm 0,03^{\mathrm{abAB}}$ \\
\hline
\end{tabular}

$\mathrm{GE}$ = gelatina $\mathrm{GE}+\mathrm{AE}=$ gelatina com ácido esteárico; $\mathrm{AM}=$ amido modificado $\mathrm{CE}=$ cera de carnaúba; $\mathrm{FO}=$ fondant $\mathrm{CO}=$ sem cobertura; e EMB = embalado. Médias seguidas de mesma letra minúscula na coluna e maiúscula na linha não diferem entre si ao nível de $5 \%$ de significância.

Tabela 6. Mastigabilidade, em g, dos bolos ao longo da estocagem.

\begin{tabular}{|c|c|c|c|c|c|c|}
\hline Tratamento & Dia 0 & Dia 1 & Dia 3 & Dia 4 & Dia 7 & Dia 10 \\
\hline $\mathrm{CO}$ & $1.592 \pm 185^{\mathrm{aC}}$ & $1.825 \pm 118^{\mathrm{aC}}$ & $1.572 \pm 220^{\mathrm{abC}}$ & $2.717 \pm 150^{\mathrm{aB}}$ & - & $3.805 \pm 376^{\mathrm{aA}}$ \\
\hline EMB & $1.592 \pm 185^{\mathrm{aA}}$ & $1.574 \pm 115^{\mathrm{abA}}$ & $1.319 \pm 178^{\mathrm{abA}}$ & $1.083 \pm 625^{\mathrm{cA}}$ & $1.183 \pm 70^{\mathrm{bA}}$ & $1.338 \pm 169^{\mathrm{bA}}$ \\
\hline GE & $1.592 \pm 185^{\mathrm{aA}}$ & $1.163 \pm 203^{\mathrm{CAB}}$ & $1.179 \pm 113^{\mathrm{bAB}}$ & $1.013 \pm 177^{\mathrm{cB}}$ & $1.419 \pm 215^{\mathrm{bAB}}$ & $1.407 \pm 477^{\mathrm{bAB}}$ \\
\hline $\mathrm{GE}+\mathrm{AE}$ & $1.592 \pm 185^{\mathrm{aA}}$ & $1.373 \pm 127^{\mathrm{bcA}}$ & $1.423 \pm 102^{\mathrm{abA}}$ & $1.244 \pm 90^{\mathrm{cA}}$ & $1.534 \pm 107^{\mathrm{bA}}$ & $1.574 \pm 360^{\mathrm{bA}}$ \\
\hline $\mathrm{AM}$ & $1.592 \pm 185^{\mathrm{aBC}}$ & $1.306 \pm 49^{\mathrm{bcc}}$ & $1.745 \pm 118^{\mathrm{aAB}}$ & $2.089 \pm 140^{\mathrm{bA}}$ & $1.638 \pm 145^{\mathrm{bBC}}$ & $2.077 \pm 405^{\mathrm{bA}}$ \\
\hline $\mathrm{CE}$ & $1.592 \pm 185^{\mathrm{aC}}$ & $1.518 \pm 108^{\mathrm{abC}}$ & $1.714 \pm 402^{\mathrm{aC}}$ & $2.140 \pm 113^{\mathrm{bBC}}$ & $2.382 \pm 970^{\mathrm{aAB}}$ & $3.267 \pm 646^{\mathrm{aA}}$ \\
\hline FO & $1.592 \pm 185^{\mathrm{aAB}}$ & $1.439 \pm 289^{\mathrm{bcAB}}$ & $1.572 \pm 220^{\mathrm{abAB}}$ & $1.302 \pm 217^{\mathrm{cB}}$ & - & $1.990 \pm 295^{\mathrm{bA}}$ \\
\hline
\end{tabular}

$\mathrm{GE}$ = gelatina $\mathrm{GE}+\mathrm{AE}=$ gelatina com ácido esteárico; $\mathrm{AM}=$ amido modificado; $\mathrm{CE}=$ cera de carnaúba; $\mathrm{FO}=$ fondant $\mathrm{CO}=$ sem cobertura; e $\mathrm{EMB}=$ embalado. Médias seguidas de mesma letra minúscula na coluna e maiúscula na linha não diferem entre si ao nível de $5 \%$ de significância. 
Tabela 7. Luminosidade $\left(L^{*}\right)$ da superfície dos bolos ao longo da estocagem.

\begin{tabular}{lcll}
\hline Tratamento & Dia 1 & Dia 3 & Dia 4 \\
\hline CO & $41,4 \pm 2,3^{\mathrm{bA}}$ & $31,2 \pm 1,7^{\mathrm{bB}}$ & $27,8 \pm 0,2^{\mathrm{bB}}$ \\
EMB & $40,3 \pm 1,2^{\mathrm{bA}}$ & $25,6 \pm 1,1^{\mathrm{cB}}$ & $27,8 \pm 0,6^{\mathrm{bB}}$ \\
GE & $39,9 \pm 0,4^{\mathrm{bA}}$ & $28,0 \pm 2,2^{\mathrm{bcB}}$ & $29,2 \pm 0,7^{\mathrm{bB}}$ \\
GE + AE & $47,5 \pm 3,3^{\mathrm{aA}}$ & $39,1 \pm 0,7^{\mathrm{aB}}$ & $41,9 \pm 3,4^{\mathrm{aAB}}$ \\
AM & $40,2 \pm 1,3^{\mathrm{bA}}$ & $30,8 \pm 1,9^{\mathrm{bB}}$ & $33,4 \pm 2,8^{\mathrm{bB}}$ \\
CE & $39,9 \pm 0,5^{\mathrm{bA}}$ & $27,9 \pm 1,2^{\mathrm{bcB}}$ & $26,4 \pm 0,9^{\mathrm{bB}}$ \\
FO & $39,6 \pm 1,3^{\mathrm{bA}}$ & $25,7 \pm 0,6^{\mathrm{cB}}$ & $25,8 \pm 0,2^{\mathrm{bB}}$
\end{tabular}

$\mathrm{GE}=$ gelatina; $\mathrm{GE}+\mathrm{AE}=$ gelatina com ácido esteárico; $\mathrm{AM}=$ amido modificado; $\mathrm{CE}=$ cera de carnaúba $\mathrm{FO}=$ fondant $; \mathrm{CO}=$ sem cobertura $; \mathrm{e} \mathrm{EMB}=$ embalado. Médias seguidas de mesma letra minúscula na coluna e maiúscula na linha não diferem entre si ao nível de $5 \%$ de significância.

Tabela 8. Croma $\left(C^{\star}\right)$ da superfície dos bolos ao longo da estocagem.

\begin{tabular}{lccc}
\hline Tratamento & Dia 1 & Dia 3 & Dia 4 \\
\hline CO & $11,77 \pm 2,08^{\mathrm{aA}}$ & $17,63 \pm 1,26^{\mathrm{aB}}$ & $15,11 \pm 1,05^{\mathrm{abAB}}$ \\
EMB & $11,92 \pm 0,12^{\mathrm{aA}}$ & $16,46 \pm 1,19^{\mathrm{aB}}$ & $15,09 \pm 0,89^{\mathrm{abB}}$ \\
GE & $6,69 \pm 0,58^{\mathrm{bcA}}$ & $10,08 \pm 1,60^{\mathrm{bcB}}$ & $10,40 \pm 0,73^{\mathrm{cdB}}$ \\
GE + AE & $10,17 \pm 0,93^{\mathrm{abA}}$ & $13,36 \pm 0,36^{\mathrm{abB}}$ & $12,60 \pm 0,12^{\mathrm{bcB}}$ \\
AM & $12,94 \pm 1,53^{\mathrm{aA}}$ & $14,04 \pm 1,93^{\mathrm{abA}}$ & $16,11 \pm 1,74^{\mathrm{aA}}$ \\
CE & $6,85 \pm 0,49^{\mathrm{bcA}}$ & $10,34 \pm 0,99^{\mathrm{bcB}}$ & $9,45 \pm 0,24^{\mathrm{dB}}$ \\
FO & $6,38 \pm 0,41^{\mathrm{cA}}$ & $8,19 \pm 1,10^{\mathrm{cA}}$ & $7,69 \pm 0,45^{\mathrm{dA}}$ \\
\hline
\end{tabular}

$\mathrm{GE}=$ gelatina; $\mathrm{GE}+\mathrm{AE}=$ gelatina com ácido esteárico; $\mathrm{AM}=$ amido modificado; $\mathrm{CE}=$ cera de carnaúba; $\mathrm{FO}=$ fondant $; \mathrm{CO}=$ sem cobertura $; \mathrm{e} \mathrm{EMB}=$ embalado. Médias seguidas de mesma letra minúscula na coluna e maiúscula na linha não diferem entre si ao nível de $5 \%$ de significância.

Tabela 9. Tom angular (h) da superfície dos bolos ao longo da estocagem.

\begin{tabular}{lccc}
\hline Tratamento & Dia 1 & Dia 3 & Dia 4 \\
\hline CO & $47,95 \pm 1,78^{\mathrm{bA}}$ & $50,88 \pm 1,86^{\mathrm{abA}}$ & $46,35 \pm 0,40^{\mathrm{bA}}$ \\
$\mathrm{EMB}$ & $47,04 \pm 0,73^{\mathrm{bA}}$ & $44,75 \pm 0,85^{\mathrm{cAB}}$ & $44,02 \pm 0,67^{\mathrm{bB}}$ \\
$\mathrm{GE}$ & $48,95 \pm 0,33^{\mathrm{bA}}$ & $45,86 \pm 0,96^{\mathrm{cA}}$ & $44,51 \pm 0,81^{\mathrm{bA}}$ \\
$\mathrm{GE}+\mathrm{AE}$ & $56,58 \pm 3,49^{\mathrm{aA}}$ & $53,61 \pm 1,66^{\mathrm{aA}}$ & $59,18 \pm 4,62^{\mathrm{aA}}$ \\
$\mathrm{AM}$ & $48,60 \pm 1,71^{\mathrm{bA}}$ & $47,25 \pm 1,37^{\mathrm{bcA}}$ & $49,13 \pm 0,82^{\mathrm{bA}}$ \\
$\mathrm{CE}$ & $48,95 \pm 0,33^{\mathrm{bA}}$ & $45,86 \pm 0,96^{\mathrm{bcB}}$ & $44,51 \pm 0,81^{\mathrm{bB}}$ \\
$\mathrm{FO}$ & $44,23 \pm 0,59^{\mathrm{bA}}$ & $36,12 \pm 1,35^{\mathrm{dB}}$ & $36,35 \pm 1,73^{\mathrm{cB}}$ \\
\hline $\mathrm{GE}=$ gelatina; GE $+\mathrm{AE}=$ gelatina com ácido esteárico; $\mathrm{AM}=$ amido modificado; \\
$\mathrm{CE}=$ cera de carnaúba; FO = fondant; CO = sem cobertura; e EMB = embalado. Médias \\
seguidas de mesma letra minúscula na coluna e maiúscula na linha não diferem entre \\
si ao nível de 5\% de significância.
\end{tabular}

Tabela 10. Diferença de cor $\left(\Delta \mathrm{E}^{*}\right)$ da superfície dos bolos ao longo da estocagem.

\begin{tabular}{lcc}
\hline Tratamento & Dia 3 & Dia 4 \\
\hline CO & $12,58 \pm 0,60^{\mathrm{abcA}}$ & $13,84 \pm 1,36^{\mathrm{aA}}$ \\
EMB & $15,42 \pm 0,65^{\mathrm{aA}}$ & $13,14 \pm 0,67^{\mathrm{aB}}$ \\
GE & $12,54 \pm 1,29^{\mathrm{abcA}}$ & $11,79 \pm 0,50^{\mathrm{abA}}$ \\
GE + AE & $9,16 \pm 2,44^{\mathrm{cA}}$ & $6,59 \pm 2,43^{\mathrm{cA}}$ \\
AM & $10,13 \pm 1,69^{\mathrm{bcA}}$ & $8,19 \pm 0,83^{\mathrm{bcA}}$ \\
CE & $12,61 \pm 0,82^{\mathrm{abcA}}$ & $13,72 \pm 0,60^{\mathrm{aA}}$ \\
FO & $14,06 \pm 0,94^{\mathrm{abA}}$ & $13,80 \pm 0,59^{\mathrm{aA}}$ \\
\hline
\end{tabular}

$\mathrm{GE}$ = gelatina; $\mathrm{GE}+\mathrm{AE}$ = gelatina com ácido esteárico; $\mathrm{AM}=$ amido modificado; $\mathrm{CE}=$ cera de carnaúba; $\mathrm{FO}=$ fondant $; \mathrm{CO}=$ sem cobertura $; \mathrm{e} \mathrm{EMB}=$ embalado. Médias seguidas de mesma letra minúscula na coluna e maiúscula na linha não diferem entre si ao nível de $5 \%$ de significância.
As coberturas à base de gelatina e de gelatina com ácido esteárico foram capazes de minimizar as alterações na atividade de água e na textura dos bolos durante a estocagem. Entretanto, a coloração da cobertura com ácido esteárico descaracterizou a aparência do bolo de chocolate. Por outro lado, os bolos com cobertura à base de gelatina apresentaram perda de massa elevada. A cobertura à base de amido foi a que melhor conservou os atributos de cor da superfície dos bolos.

Os bolos com coberturas de alto teor de umidade mostraram perda de massa superior aos bolos sem cobertura e, ao mesmo tempo, tiveram os maiores valores de atividade de água, com exceção da cobertura à base de emulsão de cera de carnaúba que foi rapidamente absorvida pelo bolo e apresentou diversas rachaduras durante a estocagem. Este comportamento, aparentemente ambíguo, pode ser atribuído à perda de umidade da cobertura para o ambiente e à diminuição da perda de umidade do miolo do bolo.

Estudos futuros podem ser conduzidos de modo a avaliar a cinética de transferência de massa (umidade) entre bolo (miolo e crosta), cobertura e ambiente, sob temperatura e umidade controladas. Além disto, a avaliação sensorial dos bolos com coberturas é um aspecto relevante a ser avaliado no desenvolvimento deste tipo de produto.

\section{Agradecimentos}

Os autores agradecem às empresas Alibra, Cargill, Emulzint, Fazendão Ovos, Gelita e Megh pela doação de matérias-primas, assim como aos funcionários da padaria pelo auxílio na parte experimental.

\section{Referências bibliográficas}

AGRITEMPO. Disponível em: <http://www.agritempo.gov.br/modules. php? name=Mapas \& estado=SP $>$. Acesso em: 24 Nov. 2005.

BALDWIN, E. A.; NISPEROS, M. O.; HAGENMAIER, R. D.; BAKER, R. A. Use of lipids in coatings for food products. Food Technology, v. 51, n. 6, p. 56-62, 64, 1997.

CEPAGRI. Publicação eletrônica [mensagem pessoal]. Mensagem recebida por<cibelec@fea.unicamp.br> em 24 nov. 2005.

CHUDZIKIEWICZ, F. F. Análise do comportamento de compra e da satisfação do cliente no mercado de panificadoras e confeitarias em Curitiba. Curitiba, 2005. 225 p. Dissertação (Mestrado em Administração) - Centro de Ciências Sociais Aplicadas, Pontifício Universidade Católica do Paraná.

COUNCIL, K. A. Analysis of variance. In: HELWIG, J. T. (Ed.). SAS Introductory guide. 3 ed. Cary: SAS Institute, 1985. p. 55-60.

CUQ, B. GONTARD, N.; CUQ, J. L.; GUILBERT, S. Selected functional properties of fish myofibrillar protein-based films as affected by hydrophilic plasticizers. Journal of Agricultural and Food Chemistry, v. 45, n. 3, p. 622-626, 1997.

CUQ, B.; GUILBERT, S. Edible film and coating as active layers. In: ROONEY, M. L. (Ed.). Active Food Packaging. London: Blackie Academic \& Professional, 1995. p. 111-142.

DENDY, D. A. V.; DOBRASZCZYK, B. J. Cereals and cereal products: chemistry and technology. Gaithersburg: Aspen, 2001.

EMULZINT. Bolomix chocolate. Disponível em: <http://www. emulzint.com.br/>. Acesso em: 24 Ago. 2005. 
GALLO, J. A. Q. DEBEAUFORT, F.; CALLEGARIN, F.; VOILLEY, A. Lipid hydrophobicity, physical state and distribution effects on the properties of emulsion- based films. Journal of Membrane Science, v. 180 , n. 1 , p. $37-46,2000$

GÉLINAS, P.; ROY, G.; GUILLET, M. Relative effects of ingredients on cake staling based on an accelerated shelf-life test. Journal of Food Science, v. 64, n. 5, p. 937-940, 1999.

GONTARD, N. Films comestibles et biodegradables: étude des propiétés filmogènes du gluten de blé. Compter rendus de l'Académie d'Agriculture de France, v. 80, n. 4, p. 109-117, 1994.

GONTARD, N. DUCHEZ, C.; CUQ, J. L.; GUILBERT, S. Edible composite films of wheat gluten and lipids: water vapour permeability and other physical properties. International Journal of Food Science and Technology, v. 29, n. 1, p. 39-50, 1994.

JONES, H. P. Ambient packaged cakes. In: MAN, C. M. D.; JONES, A. A. (Eds.). Shelf life evaluation of foods. London: Chapman \& Hall, 1996. p.179-201.

KESTER, J. J.; FENNEMA, O. R. Edible films and coatings: a review. Food Technology, v. 40, n. 12, p. 47-59, 1986.

KOELSCH, C. M.; LABUZA, T. P. Functional, physical and morphological properties of methyl cellulose and fatty acid-based edible barriers. Lebensmittel Wissenschaft und Technologie, v. 25, n. 5, p. 404-411, 1992.

KROCHTA, J. M.; MULDER-JOHNSTON, C. Edible and biodegradable polymer films: challenges and opportunities. Food Technology, v. 51, n. 2 , p. $61-74,1997$.

MEG. Frutas. Disponível em:<http://www.megh.com.br/index_03_03. php >. Acesso em: 30 set. 2005.

MINOLTA. Precise color communication: color control from feeling to instrumentation. Osaka, 1994.
PAVANELLI, A. P.; CICHELLO, M. S.; PALMA, E. J. Emulsificantes como agentes de aeração em bolos. Disponível em: $<\mathrm{http}: / / w w w$. oxiteno.com.br>. Acesso em: 12 Dez. 2000.

PÉROVAL, C. DEBEAUFORT, F.; DESPRÉ, D.; VOILLEY, A. Edible arabinoxylan-based films. 1. Effects of lipid type and water vapor permeability, film structure, and other physical characteristics. Journal of Agricultural and Food Chemistry, v. 50, n. 14, p. 3977-3983, 2002.

POTTER, N. N.; HOTCHKISS, J. H. Food science. 5 ed. Gaithersburg: Aspen, 1998.

SARANTÓPOULOS, C. I. G. L. OLIVEIRA, L. M.; PADULA, M.; COLTRO, L.; ALVES, R. M. V.; GARCIA, E. E. C. Embalagens plásticas flexíveis: principais polímeros e avaliação de propriedades. Campinas: CETEA/ITAL, 2002.

SARMIENTO-CONSOLE, F. M. Z. Otimização das condições de hidrólise ácida do amido de mandioca para obtenção de substituto de gordura: caracterização de hidrolisados e aplicação em bolos. Campinas, 1998. 155p. Tese (Doutorado em Tecnologia de Alimentos) - Faculdade de Engenharia de Alimentos, Universidade Estadual de Campinas - UNICAMP.

SLUIMER, P. Principles of breadmaking: functionality of raw materials and process steps. Minnesota: AACC, 2005.

WONG, D. W. S. GASTINEAU, F. A.; GREGORSKI, S. S.; TILLIN, S. J.; PAVLATH, A. E. Chitosan-lipid films: microstructure and surface energy. Journal of Agricultural and Food Chemistry, v. 40, n. 4, p. 540-544, 1992.

YANG, L.; PAULSON, A. T. Effects of lipids on mechanical and moisture barrier properties of edible gellan film. Food Research International, v. 33, n. 7, p. 571-578, 2000. 